\title{
Fast wave propagation in auditory cortex of an awake cat using a chronic microelectrode array*
}

\author{
Russell S Witte $^{1}$, Patrick J Rousche ${ }^{2}$ and Daryl R Kipke ${ }^{1}$ \\ ${ }^{1}$ Biomedical Engineering, University of Michigan, Ann Arbor, MI, USA \\ ${ }^{2}$ Biomedical Engineering, University of Illinois at Chicago, Chicago, IL, USA \\ E-mail: ruswit@umich.edu
}

Received 29 November 2006

Accepted for publication 9 January 2007

Published 28 February 2007

Online at stacks.iop.org/JNE/4/68

\begin{abstract}
We investigated fast wave propagation in auditory cortex of an alert cat using a chronically implanted microelectrode array. A custom, real-time imaging template exhibited wave dynamics within the 33-microwire array $\left(3 \mathrm{~mm}^{2}\right)$ during ten recording sessions spanning 1 month post implant. Images were based on the spatial arrangement of peri-stimulus time histograms at each recording site in response to auditory stimuli consisting of tone pips between 1 and $10 \mathrm{kHz}$ at $75 \mathrm{~dB}$ SPL. Functional images portray stimulus-locked spiking activity and exhibit waves of excitation and inhibition that evolve during the onset, sustained and offset period of the tones. In response to $5 \mathrm{kHz}$, for example, peak excitation occurred at $27 \mathrm{~ms}$ after onset and again at $15 \mathrm{~ms}$ following tone offset. Variability of the position of the centroid of excitation during ten recording sessions reached a minimum at $31 \mathrm{~ms}$ post onset $(\sigma=125 \mu \mathrm{m})$ and $18 \mathrm{~ms}$ post offset $(\sigma=145 \mu \mathrm{m})$, suggesting a fine place/time

representation of the stimulus in the cortex. The dynamics of these fast waves also depended on stimulus frequency, likely reflecting the tonotopicity in auditory cortex projected from the cochlea. Peak wave velocities of $0.2 \mathrm{~m} \mathrm{~s}^{-1}$ were also consistent with those purported across horizontal layers of cat visual cortex. The fine resolution offered by microimaging may be critical for delivering optimal coding strategies used with an auditory prosthesis. Based on the initial results, future studies seek to determine the relevance of these waves to sensory perception and behavior.
\end{abstract}

M] This article features online multimedia enhancements

(Some figures in this article are in colour only in the electronic version)

\section{Introduction}

A 'click' sound launches a cascade of rapid mechanical and electrical events that extend from the cochlea and radiate through the intricate central auditory pathway to the cerebral cortex. Auditory perception emerges from this dynamic ensemble of neuronal activity distributed across the cortex.

* The work was performed at Department of Bioengineering, Arizona State University, ECG 334 MS-9709 Arizona State University, Tempe, AZ 852879709, USA.
In 1927, Ivan Pavlov speculated that the cortex operated as a 'rich mosaic' composed of localized functional regions of excitation and inhibition (Pavlov 1927). A generation later, Lilly first identified spatial patterns of electrocortical activity in single trials and referred to them as 'figures' moving in time and space across the cortical surface (Lilly and Cherry 1954). There is some evidence that propagating waves contribute to the sensory experience, but the spatial and temporal properties of these waves, and their relevance in the cortex, remain unclear (Krupa et al 2004). The organization 
of the auditory system and inferences from psychophysical studies suggest that dynamic processing evolves on the order of microns and milliseconds. The human cochlea, for instance, encodes sound frequency based on the tonotopic gradient of 0.2 oct $\mathrm{mm}^{-1}$ (Greenwood 1990). Moreover, the cochlea's traveling wave can take $10 \mathrm{~ms}$ or longer to propagate from base to apex (Ruggero and Rich 1987, Goldstein et al 1971). These parameters provide a physiologic baseline for the speed, latency and spatial acuity of the sound-encoding organ that feeds the auditory nerve. In the psychophysical world, a delay as short as $20 \mathrm{~ms}$ between speech sound and lip reading affects comprehension (Summerfield 1992). Based in part on these simple examples, we hypothesized that cortical processing and auditory perception also evolve on a critical scale of microns and milliseconds.

Despite the revolution of modern brain imaging that followed Lilly's initial observations, detecting waves of cortical activity in response to stimuli or behavior with fine spatial and temporal precision remains especially challenging. Noninvasive imaging, such as PET and fMRI, rely on slow metabolic signals loosely correlated with electrical activity. Although electroencephalography (EEG) and magnetoencephalography (MEG) depict electric or magnetic dipole fields directly linked to synchronous neuronal activity, the best spatial resolution of a combined system is on the order of a centimeter.

Whereas noninvasive functional brain imaging provides gross information regarding the underlying neural activity across a large region, invasive methods enable tracking a small population of neurons and detecting fast signals directly linked to action potentials. Optical imaging using a voltage sensitive dye (VSD), for instance, provides valuable information related to membrane potential propagation and underlying neural connectivity in response to sensory stimuli and behavior (Contreras and Llinas 2001, Briggman et al 2005). Because optical imaging with VSDs involves measuring small differential signals in an exposed setting ( $<0.5 \%$ per $100 \mathrm{mV}$ change in absorbance or fluorescence), the technique is generally unsuitable for chronic recording from awake, behaving animals (exception, (Ferezou et al 2006)). Penetrating high-impedance electrodes, on the other hand, detect action potentials near the recording site and are routinely used in awake animals. When electrodes are assembled into a large array, they provide additional spatial information related to the array geometry and density of recording sites. Two recent studies revealed tonotopic organization in cat auditory cortex using microelectrode arrays (Kim et al 2006, Witte and Kipke 2005). In an effort to capture fast cortical waves in response to acoustic stimuli, we implanted a chronic rectangular array of microwires in the auditory cortex of a cat. A novel, real-time imaging template helped visualize space-time dynamics of excitatory and inhibitory waves in response to simple sounds. Spatiotemporal patterns likely reflect a combination of the neural architecture of the cortex, characteristics of the underlying auditory structures and properties of acoustic stimulation, all of which may contribute to sensory perception and decision making.
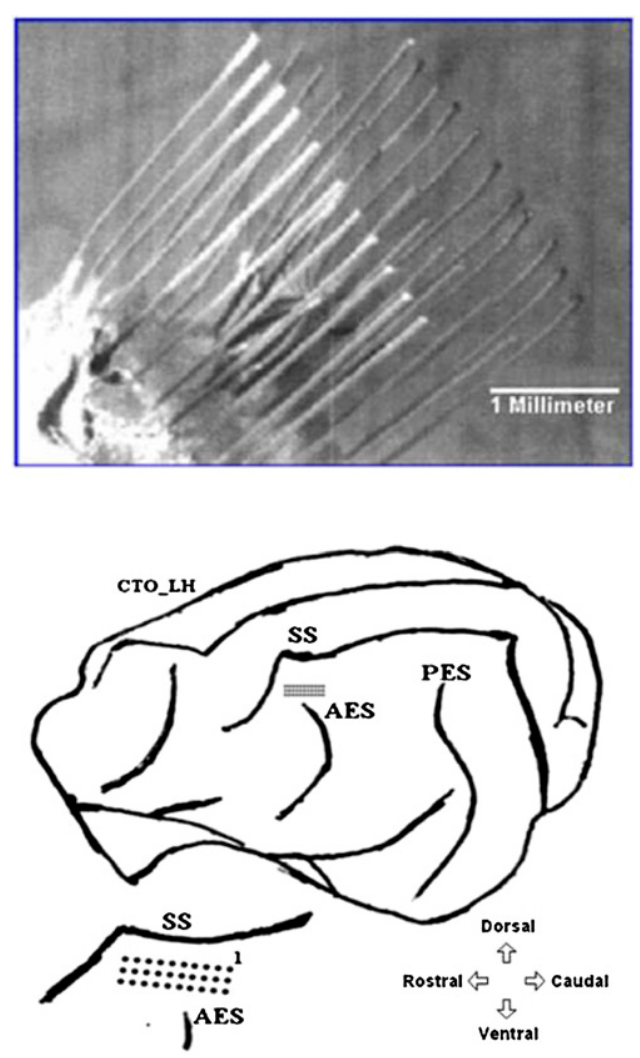

Figure 1. Top: stereoscopic photograph of the chronic tungsten microelectrode array used to capture neural responses from cat auditory cortex. The array dimensions were approximately 1 by $3 \mathrm{~mm}$ and contained 33 tungsten microelectrode wires $(500 \mu \mathrm{m}$ between rows and $300 \mu \mathrm{m}$ between columns). The $50 \mu \mathrm{m}$ diameter wires were insulated with Teflon ${ }^{\mathrm{TM}}$ and cut blunt at the tip. Bottom: location of the electrode array in cat auditory cortex. Anatomical landmarks close to the implant site and frequency response properties of neural signals indicate this implant was in the anterior auditory field (AAF). Further details of the surgical procedure and frequency response properties of this implant are reported in Witte and Kipke (2005).

\section{Methods}

\section{Surgical implant}

All experimental procedures and animal care were carried out in compliance with guidelines established by the National Institutes of Health and were approved by the Institutional Animal Care and Use Committee. One adult female cat (Felis catus) with normal hearing was implanted with a fixed electrode array $\left(3 \mathrm{~mm}^{2}\right)$ of 33 polyimide-coated tungsten microwires ( 3 rows of 11 microwires each) with 30 functional electrodes (three electrodes were reserved for grounds). The spacing was $500 \mu \mathrm{m}$ and $300 \mu \mathrm{m}$ between rows and columns, respectively. A stereoscope photograph of the array and location in cat auditory cortex are illustrated in figure 1 .

The surgical procedure has been previously described and will only be summarized (Witte and Kipke 2005, Williams et al 1999). The $50 \mu \mathrm{m}$ diameter wires were blunted and exposed at the tip. Anesthesia was administered with an initial injection of $60 \mathrm{mg}$ of ketamine in $0.7 \mathrm{ml}$ saline and maintained with isofluorane. Heart rate and saturated oxygen 
per cent were monitored throughout the procedure. Stainless steel bone screws were used to provide electrode stability and grounding. A craniotomy was performed using an $8 \mathrm{~mm}$ trephine, followed by removal of dura mater. Placement of the craniotomy was based primarily on expected stereotactic coordinates of cat auditory cortex with special care to avoid rupturing visible superficial blood vessels. The array was positioned perpendicular to the auditory cortex and advanced slowly to penetrate pia mater. Using a $200 \mu \mathrm{m}$ down, $100 \mu \mathrm{m}$ up insertion technique, electrode tips were advanced approximately $1.0 \mathrm{~mm}$ below the brain surface. The final array location resided in the middle layers of the anterior auditory field (AAF), deduced from stereotactic coordinates, receptive field properties of the recorded neural population, and anatomical landmarks near the implant site.

\section{Electrophysiology and auditory stimulation}

Neural data were obtained using a multichannel neural recording system (Plexon Inc., Dallas, TX) that enabled online and offline spike classification and sorting. Each active channel typically captured isolated waveforms composed of one to five single neurons. An alert cat was placed in an anechoic sound chamber and randomly presented 20 repetitions of 60 different frequencies ranging from 1 to $10 \mathrm{kHz}$ at a single intensity (75 dB SPL) using a calibrated auditory stimulation system (Tucker Davis Technologies, Gainesville, FL). These stimuli (200 ms on/500 ms off, $10 \mathrm{~ms}$ rise/fall) were used to examine the spatial representation of sound frequency in the cortex and determine receptive fields at each electrode. The cat was restrained using a sling, and the head was not fixed. Further details on neural recording and auditory stimulation have been previously reported (Witte and Kipke 2005). Standard estimates of best frequency (BF) at each recording site were based on the sound frequency that elicited a maximum response within the first $50 \mathrm{~ms}$ after tone onset. Spiking activity from ten recording sessions spanning 25 days were used to calculate receptive fields and generate microimages that portray dynamic spatiotemporal patterns.

\section{Generation of microimages}

Microimages were based on the spatial distribution of peristimulus time histograms, called super PSTHs, which were calculated for each electrode in response to the auditory stimuli. The custom visualization template is based on our previously published work (Witte and Kipke 2001, Witte 2002). Spike counts per bin were converted to standard deviations over the mean background rate ( $Z$ scores), obtained during $50 \mathrm{~ms}$ preceding the stimuli. Pixels were intensity coded such that gray reflects firing rates similar to background $(p>0.05)$, dark tones denote inhibition and bright tones represent excitation. Thus, a pixel in the raw microimage represents a change in spike rate within the detection region of the recording site relative to its background firing rate and scaled according to probability using a $Z$ score transformation. Such standardization across recording sites is commonplace for both microelectrode and EEG studies.
The dimensions of a microimage are determined by the size and shape of the electrode array. A variety of electrode array geometries are described in a recent review chapter (Schmidt 1999). Each recording site, $n$, contributes a pixel $\left(I_{n}\right)$, assuming that a neural signal can be discriminated from background noise. Figure 2(a) illustrates the formulation of a microimage and the standard numbering convention for a rectangular array of electrodes ( $x$ columns by $y$ rows), such as the tungsten microwire array used in this study.

A site is considered responsive to a stimulus when its neural signal elicits a time-locked response to repeated presentations of the stimulus. The diameter of each pixel (figure 2(a)) corresponds to the electrode detection region, which is related to several factors, such as cell size and electrode impedance. Given a single monopolar spherical electrode in a quasi-static isotropic medium, the extracellular potential dissipates by a factor of $1 / r$, where $r$ is the distance from the source (Rattay 1989, Humphrey 1979). Electrode impedances, which were on the order of $200 \mathrm{k} \Omega$ at $1 \mathrm{kHz}$, can actually vary during the lifetime of the implant, depending on the electrode type, degree of micromotion and tissue response to the implant (Williams 2001, Ludwig et al 2006). Because impedances were not continuously monitored and the exact location of the cells were not known, we estimated the recording detection window of each electrode (i.e., image pixel size) to be fixed at $100 \mu \mathrm{m}$ based on the physical properties of the microwires and estimates from previous studies (Buzsaki 2004). Typically, at distances greater than $100 \mu \mathrm{m}$ from the tip, only local field potentials are detectable. Note that the relative position and spacing of the electrodes are crucial for interpreting the spatiotemporal patterns inherent in the microimages and not necessarily the actual size of the pixel. If an electrode was unresponsive $(\mathrm{SNR}<2)$ for a particular recording session, an interpolated estimate was obtained based on responses at neighboring recording sites and weighted according to their distance from the unresponsive electrode.

The density of recording sites ultimately determines the maximum spatial resolution of the microimage. A single microelectrode is usually capable of detecting action potentials from multiple cells. The exact location of each cell, however, is typically not known. Therefore, to avoid spatial ambiguity, all spikes detected on the same electrode were combined to form a multi-unit response (MU) composed of approximately one to five neurons. The sampling rate of the recording system determines maximal temporal resolution. The neural recording system simultaneously sampled at $40 \mathrm{kHz}$ per channel, more than sufficient for resolving single action potentials. The chosen bin size of the PSTHs also affects the temporal resolution of the image. Sufficient sampling of neural responses is required such that the observed spike count in each bin accurately reflects the driven firing rate and approximates the probability distribution for the time frame. The noise associated with this estimate decreases by approximately the square root of the number of stimulus repetitions, but increases at the same rate with smaller bin sizes. A balance must be established, therefore, between stimulus repetition, bin size and assumptions of stationarity such that a microimage reflects the desired temporal resolution. For this study, super PSTHs 


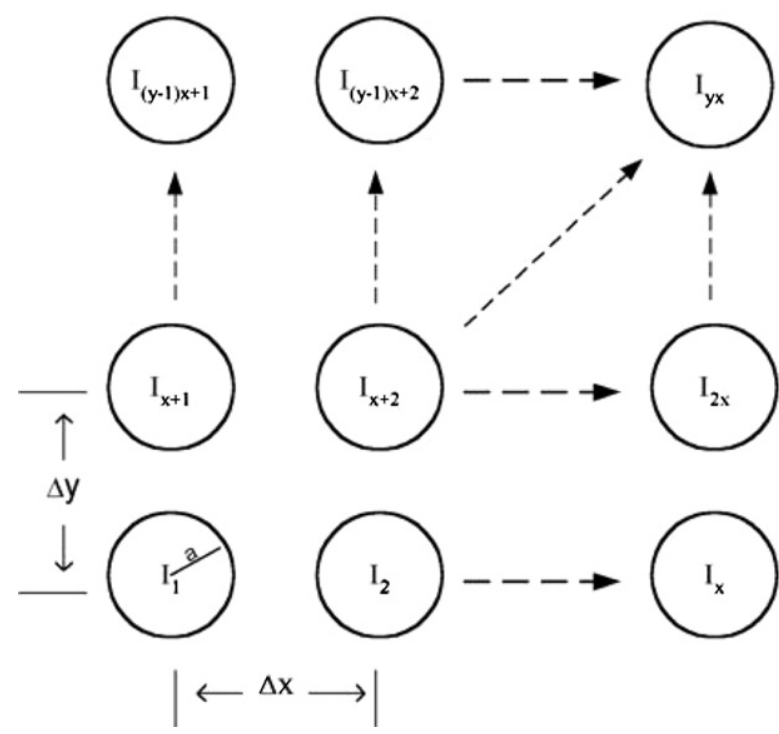

(a)

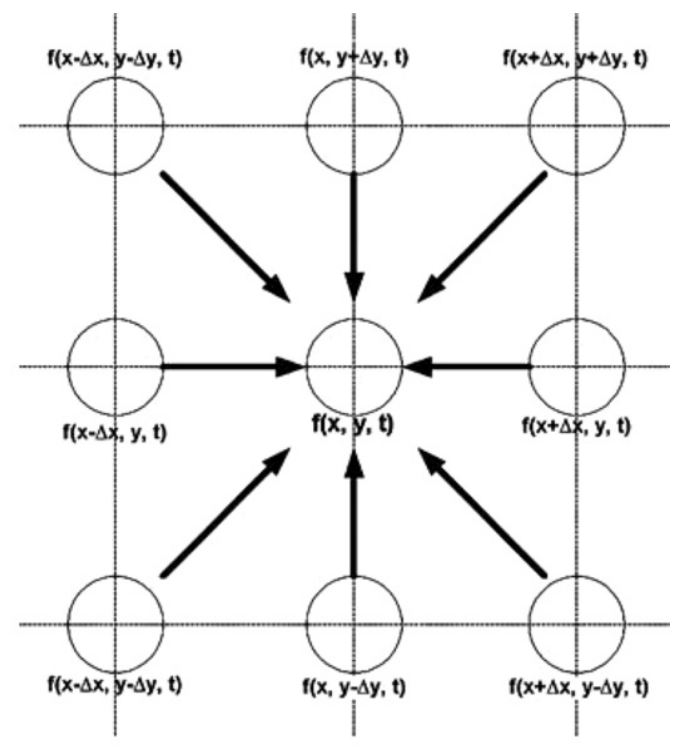

(b)

Figure 2. (a) Formulation of a microimage using a rectangular array of electrodes ( $x$ columns by $y$ rows), such as the tungsten microwire array. The subscripts indicate the numbering convention of the electrodes based on $x$ and $y$. Each recording site $(n)$ contributes a pixel magnitude $\left(I_{n}\right)$, assuming that a neural signal can be discriminated from background noise. A site is considered responsive to a stimulus when its neural signal elicits a time-locked response to repeated presentations of the stimulus. The diameter of each pixel (a) relates to the detection field of each recording site, which was estimated to be $100 \mu \mathrm{m}$ in diameter (see text for details). (b) Configuration for a single element determined from the discrete version of the GVFs is depicted in (a). The arrows by convention point towards excitation. GVFs are not as susceptible to edge effects as the centroid representation and can identify channels of excitation or inhibition.

with $1 \mathrm{~ms}$ bins were convolved with a Gaussian smoothing kernel ( $\sigma=2 \mathrm{~ms}$ ) to eliminate edge effects associated with square bins. Finally, microimages were generated in a custom data analysis and graphic software package written in Matlab 7.0 $0^{\mathrm{TM}}$ (Mathworks, Inc.). The program requires, in particular, the spacing of the recording sites, array geometry and timebinned responses to an external event.

\section{Tracking flow of excitation and inhibition}

Centroids and gradient vector fields were used to depict spatial and temporal patterns deduced from the microimages. Centroids represented the magnitude and position of excitation and inhibition at each time point, determined from the following equation:

$$
\left(C_{x}, C_{y}\right)=\left(\frac{\sum_{i=1}^{n} X_{i} W_{i}}{\sum_{i=1}^{n} W_{i}}, \frac{\sum_{i=1}^{n} Y_{i} W_{i}}{\sum_{i=1}^{n} W_{i}}\right)
$$

where $\left(X_{i}, Y_{i}\right)$ and $W_{i}$ are the coordinates and weight ( $Z$ score) of the $i$ th recording site, respectively. Sites with positive $Z$ scores were extracted to calculate an excitatory centroid (visualized as a yellow circle in the background of each microimage), whereas sites with negative $Z$ scores were used to calculate an inhibitory centroid for each frame of the microimage (visualized as a cyan circle). For each time point, $Z$ scores were summed across the array and converted to a new $Z$ score based on observations during the pre-stimulus time. The absolute value of the new $Z$ score represented the magnitude of the centroid and diameter of the plotted circle. Centroid velocity was determined by calculating its change in position during a time sequence of images. Although the centroid position may be particularly sensitive to image edge effects, the size of the centroid refers to the population response, which is independent of its spatial origin. The microimages also describe directional excitation and inhibition, such as anterior to posterior or dorsal to ventral, using a summed projection to each axis. For each time frame, the $\mathrm{Z}$ scores of all electrodes in each row (or column) are combined. The mean value is converted to a new $\mathrm{Z}$ score relative to values computed during the background window.

In addition to centroid and axes projections, images incorporated gradient vector fields (GVF) to track excitation flow. A discrete version of the following 2D model equation was used to approximate the $2 \mathrm{D} \mathrm{GVF}$ at each time frame:

$$
\nabla F(x, y)=F_{x}+F_{y},
$$

where $F(x, y)$ represents a space varying function, such as the firing rate, and $F_{x}+F_{y}$ are spatial derivatives. The configuration for a single element is depicted in figure 2(b), with arrows pointing towards excitation by convention; that is, a positive spatial gradient indicates a flow from less excited to more exited cells based on the standardized firing rates in the local region. These plots have particular advantage over the centroid calculation when an image is composed of local regions of excitation and inhibition, a more common event as the number of recording sites and spatial scope of the array increases. Also, because GVFs are not as susceptible to edge effects as centroids, they provide a more complete description of the excitation flow through a patch of cortex. These image processing techniques were used to portray waves of neural activity and quantify flow of excitation and inhibition in response to sounds presented to the cat. 

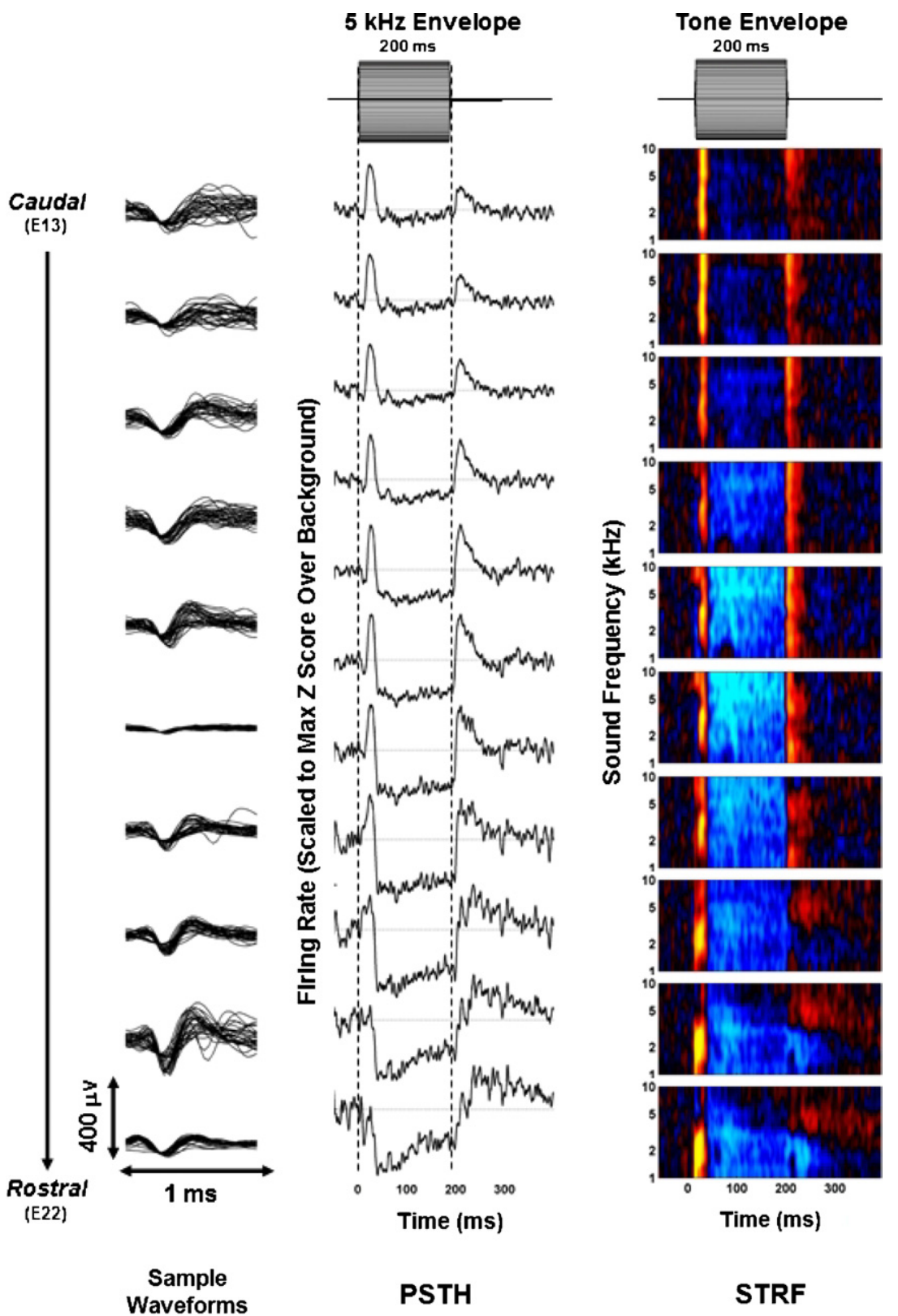

Figure 3. A sample of 30 waveforms ( $400 \mu \mathrm{v}$ full scale), PSTHs and spatiotemporal receptive fields (STRFs) for the entire middle row of the electrode array (arranged vertically from caudal to rostral). The peak amplitude of these waveforms ranged from 60 to $400 \mu \mathrm{V}$. Thresholds were set to at least twice the level of background noise (SNR $\geqslant 2$ ). The PSTHs in response to $5 \mathrm{kHz}$ tones and STRFs were scaled to the maximum $Z$ score, denoting relative firing rate compared to the background period.The STRFs exhibit significant onset and offset excitation (hot colors) and sustained inhibition (cold colors) within the displayed $450 \mathrm{~ms}$ window for frequencies between 1 and $10 \mathrm{kHz}$. Note that the most rostral sites have the strongest early onset response to low frequencies $(<5 \mathrm{kHz})$, consistent with the tonotopic organization of AAF in the cat. It should also be mentioned that both suppressive and refractory effects may contribute to overall inhibition.

\section{Results}

Neural activity on 29 of 30 electrodes (97\%) had a significant response to the sound stimuli. Figure 3 offers a sample of 30 waveforms (column 1), PSTHs (column 2) and spatiotemporal receptive fields (STRFs, column 3) for the entire middle row of the electrode array arranged vertically for display purposes (400 $\mu \mathrm{v}$ full scale). The peak amplitude of these sample waveforms ranged from 60 to $400 \mu \mathrm{V}$. Thresholds were set to at least twice the level of background noise (SNR $\geqslant 2$ ). The STRFs exhibit significant onset and offset excitation (hot colors) and sustained inhibition (cold colors) within the displayed $450 \mathrm{~ms}$ window for frequencies between 1 and $10 \mathrm{kHz}$. Note that the most rostral sites have the strongest early onset response to low frequencies $(<5 \mathrm{kHz})$, consistent with the tonotopic organization of AAF in the cat. It should also be mentioned that both suppressive and refractory effects may contribute to overall inhibition.

Figure 4 illustrates the custom microimaging template for a single frequency used to visualize evolving neural activity. The top row depicts PSTHs to the $5 \mathrm{kHz}$ tones for the active recording sites (average for the ten recording sessions, 


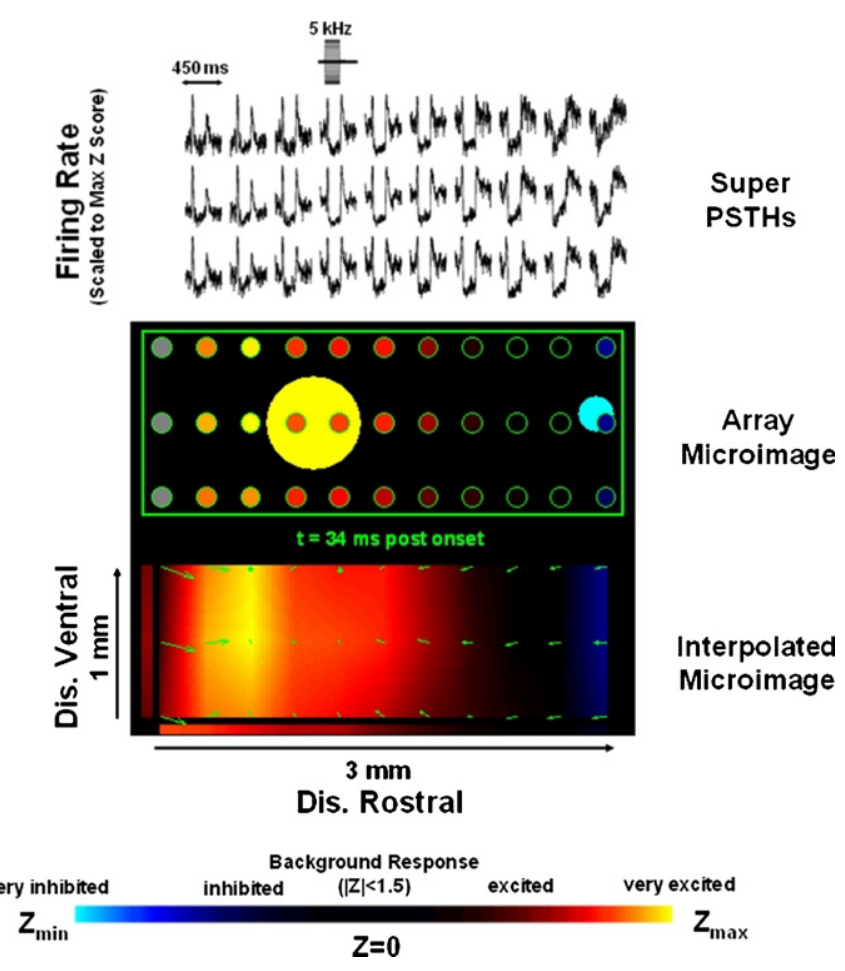

Figure 4. Custom microimaging template for a single frequency used to visualize evolving neural activity. Top row: PSTHs to the $5 \mathrm{kHz}$ tones for the active recording sites (average for the ten recording sessions, 200 total presentations). Super PSTHs with $1 \mathrm{~ms}$ bins are scaled to their peak $Z$ score and spatially arranged according to the relative orientation of the recording sites. A portion of the background response used to calculate $Z$ scores is shown before stimulus onset. The timescale for each PSTH extends for $450 \mathrm{~ms}$, starting at $50 \mathrm{~ms}$ before tone onset. The middle row represents a raw microimage (at a single time point, $34 \mathrm{~ms}$ following tone onset) with the intensity of each pixel indicating the firing rate relative to the background period. The larger filled circles denote the excitatory $\left(\mathrm{C}_{\mathrm{Exc}}\right.$, yellow) and inhibitory centroids $\left(\mathrm{C}_{\mathrm{Inh}}\right.$, cyan), with the size reflecting their magnitude. The green frame indicates that the stimulus is playing. The bottom row of figure 4 represents a spatial linear interpolation of the raw microimage after applying a Gaussian filter $(\sigma=300 \mu \mathrm{m})$. The spatial gradient vector field is superimposed on the interpolated image with each arrow indicating direction and magnitude of flow of excitation (i.e., regions of increased firing rate). Average magnitude projected along the dorsal-ventral and rostral-caudal axes is also illustrated at the left and bottom boundaries.

200 total presentations). Super PSTHs with $1 \mathrm{~ms}$ bins are scaled to their peak $Z$ score and spatially arranged according to the relative orientation of the recording sites. A portion of the background response used to calculate $Z$ scores is shown before stimulus onset. The timescale for each PSTH extends for $450 \mathrm{~ms}$, starting at $50 \mathrm{~ms}$ before tone onset. The middle row represents a raw microimage (at a single time point, $34 \mathrm{~ms}$ following tone onset) with the intensity of each pixel indicating the firing rate relative to the background period. The larger filled circles denote the excitatory $\left(\mathrm{C}_{\mathrm{Exc}}\right.$, yellow) and inhibitory centroids $\left(\mathrm{C}_{\text {Inh }}\right.$, cyan), with the size reflecting their magnitude. The green frame indicates that the stimulus is playing. The bottom row of figure 4 represents a spatial linear interpolation of the raw microimage after applying a Gaussian filter $(\sigma=$ $300 \mu \mathrm{m})$. The spatial gradient vector field is superimposed

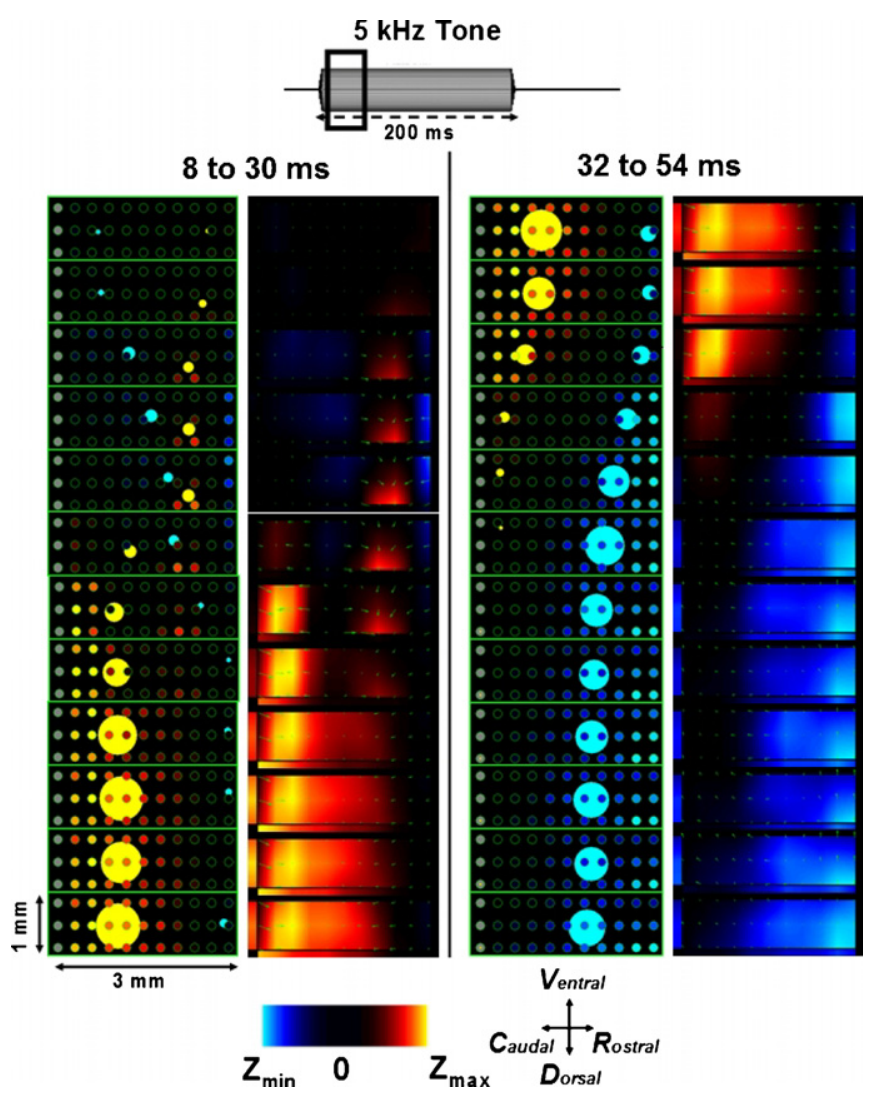

Figure 5. A time-sequenced set of microimages depict a portion of the onset responses to $5 \mathrm{kHz}$ tones ( $2 \mathrm{~ms}$ per frame, spanning 8 to $54 \mathrm{~ms}$ after tone onset). An initial excitatory wave begins rostral at $t=10 \mathrm{~ms}$, peaks at $t=28 \mathrm{~ms}$, and disappears by $40 \mathrm{~ms}$, when an inhibitory wave first appears. The images correspond with the super PSTHs presented in figure 4.

M A GIF movie of this figure is available from stacks.iop.org/JNE/4/68

on the interpolated image with each arrow indicating direction and magnitude of flow of excitation (i.e., regions of increased firing rate). Average magnitude projected along the dorsalventral and rostral-caudal axes is also illustrated at the left and bottom boundaries.

A time-sequenced set of microimages depict a portion of the onset responses to $5 \mathrm{kHz}$ tones in figure 5 ( $2 \mathrm{~ms}$ per frame, spanning 8 to $54 \mathrm{~ms}$ after tone onset). An initial excitatory wave begins rostral at $t=10 \mathrm{~ms}$, peaks at $t=28 \mathrm{~ms}$, and disappears by $40 \mathrm{~ms}$, when an inhibitory wave first appears. The images correspond with the super PSTHs presented in figure 4.

A procession of excitatory (yellow) and inhibitory (cyan) centroids portray waves of cortical activity. A multimedia movie illustrates the complete wave pattern associated with figure 5 spanning $350 \mathrm{~ms}$ (starting $10 \mathrm{~ms}$ before stimulus onset). Figure 6 (top) tracks the position of these centroids along the rostral-caudal axis during the $5 \mathrm{kHz}$ stimulus, with the size of the symbol denoting relative centroid magnitude (open circles, $Z \geqslant 3$ ). Note that at certain times, excitation and suppression appear simultaneously within the array, although at different positions. For this stimulus, peak excitation occurred at $27 \mathrm{~ms}$ after onset and again at $15 \mathrm{~ms}$ following 


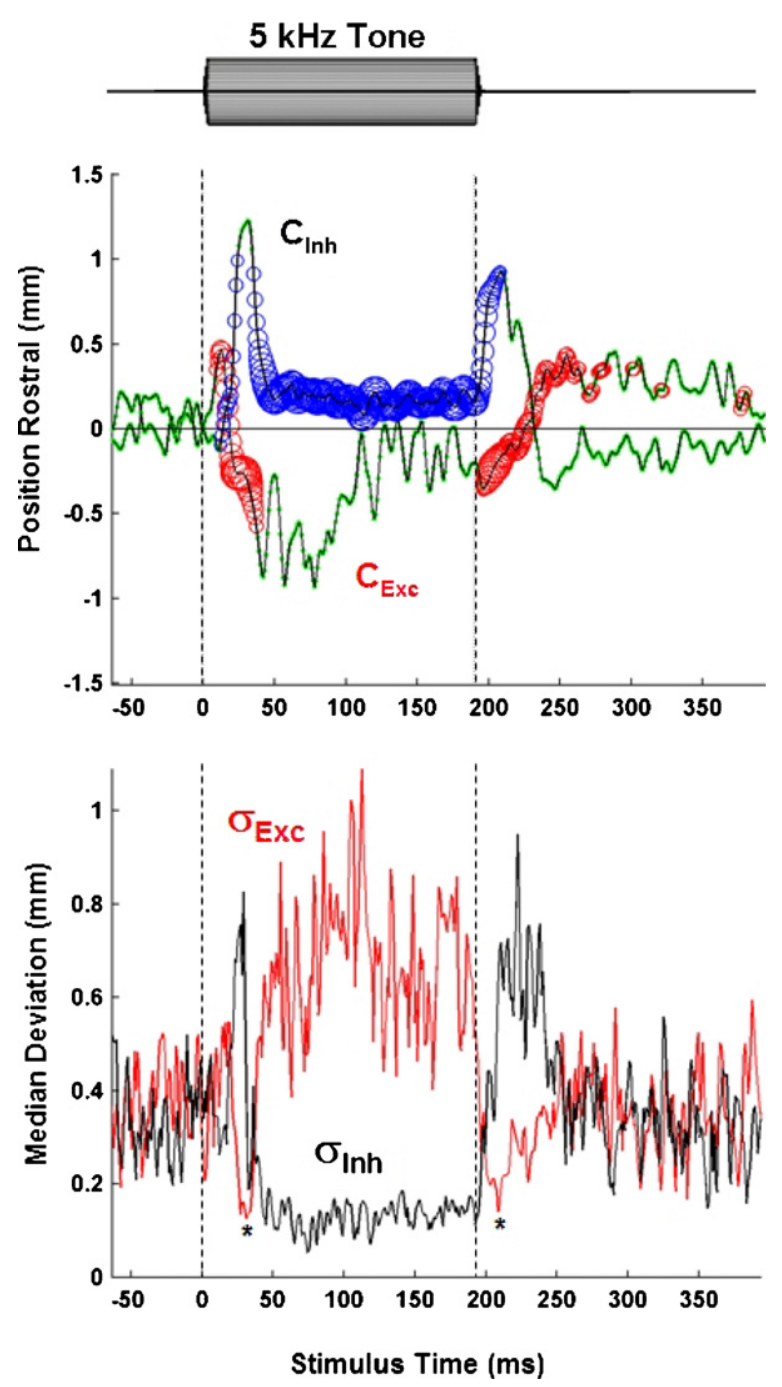

Figure 6. Top: position of $\mathrm{C}_{\mathrm{Exc}}$ and $\mathrm{C}_{\text {Inh }}$ along the rostral-caudal axis during the $5 \mathrm{kHz}$ stimulus, with the size of the symbol denoting relative centroid magnitude (all open circles, $Z \geqslant 3$ ). Note that at certain times, excitation and suppression appear simultaneously within the array, although at different positions. For this stimulus, peak excitation occurred at $27 \mathrm{~ms}$ after onset and again at $15 \mathrm{~ms}$ following tone offset. Bottom: The median deviation for all centroid positions (light red $=$ excitatory centroid) at $5 \mathrm{kHz}$ spanning the ten recording sessions ( 25 days). Variability of the position of $\mathrm{C}_{\mathrm{Exc}}$ during ten recording sessions reached a minimum at $31 \mathrm{~ms}$ post onset $(\sigma=125 \mu \mathrm{m})$ and $18 \mathrm{~ms}$ post offset $(\sigma=145 \mu \mathrm{m})$. These points are marked with an asterisk. Deviations as small as $100 \mu \mathrm{m}$ during the onset, sustained and offset intervals reflect transient periods of fine space/time cortical registration of the stimuli.

tone offset. The variability of the $\mathrm{C}_{\mathrm{Exc}}$ position during ten recording sessions reached a minimum at $31 \mathrm{~ms}$ post onset $(\sigma=125 \mu \mathrm{m})$ and $18 \mathrm{~ms}$ post offset $(\sigma=145 \mu \mathrm{m})$.

Figure 6 (bottom) depicts the median deviation for all centroid positions (red and black lines refer to $\mathrm{C}_{\mathrm{Exc}}$ and $\mathrm{C}_{\mathrm{Inh}}$, respectively) at $5 \mathrm{kHz}$ spanning the ten recording sessions. Deviations as small as $100 \mu \mathrm{m}$ during the onset, sustained and offset intervals reflect transient periods of fine space/time cortical registration of the stimuli.

We also examined the dependence of sound frequency on these waves. Response properties such as best frequency
(BF) or characteristic frequency (CF) are commonly used to characterize a neuron's stimulus preference. Because the intensity of these simuli was fixed at $75 \mathrm{~dB}$ SPL, we accordingly compared wave activity to a BF calculation at each position along the caudal-rostral axis for tones between 1 and $10 \mathrm{kHz}$ at $75 \mathrm{~dB}$ SPL. The BF was defined as the frequency that elicited the largest response based on the mean firing rate during the initial $50 \mathrm{~ms}$ after tone onset. For this initial study in AAF, a tonotopic arrangement for the most rostral recording sites $\left(-0.94\right.$ oct $\left.\mathrm{mm}^{-1}, r^{2}=0.91\right)$ is evident in figure $7(\mathrm{a})$, with error bars indicating standard error (SE). A tonotopic arrangement is also evident in both the inhibitory and excitatory centroids at specific time points (17 and $35 \mathrm{~ms}$ ) in figures 7(b) and 7(c), with horizontal error bars denoting SE. The tonotopic gradient based on $\mathrm{C}_{\mathrm{Exc}}$ at $17 \mathrm{~ms}$ was -3.3 oct $\mathrm{mm}^{-1}\left(r^{2}=0.96\right)$; if the spatial peak is used, rather than centroid, the gradient reduces to 1.6 oct $\mathrm{mm}^{-1}$, but with a smaller correlation coefficient $\left(r^{2}=0.79\right)$. The gradient is similar $18 \mathrm{~ms}$ later $\left(-3.4\right.$ oct mm $\left.\mathrm{mm}^{-1}\right)$, but the excited region is shifted approximately $5 \mathrm{~mm}$ caudally. Inhibitory regions also appear to maintain a gradient at these time points, suggesting that both excitation and suppression may contribute to encoding sound frequency. Note for consistent comparison, the abscissa and ordinate are the same as in figure 7(a), even though the dependent and independent variables are reversed for the regression analysis. The reported slope, therefore, is the inverted slope from the linear regression. These results indicate that waves of activity sweep across the cortex on the order of microns and milliseconds and depend on features of the stimuli. Moreover, they can occur in a much smaller bin size than that typically used to measure a neuron's response property (e.g., $50 \mathrm{~ms}$ ).

Not only did the position of excitation and inhibition correlate with sound frequency, but the instantaneous velocity also varied systematically with the stimulus. The frequency/velocity relationship for $\mathrm{C}_{\mathrm{Exc}}$ at three time points is conveyed in figure 8 , with a gradient peaking at $10 \mathrm{~ms}$ after stimulus onset $\left(m=-0.05\left(\mathrm{~m} \mathrm{~s}^{-1}\right) / \mathrm{oct}, r^{2}=0.77\right)$. The magnitude of $\mathrm{C}_{\mathrm{Exc}}$ was significant across all frequencies at these times (all open circles, $Z \geqslant 3$ ). Diameter of the symbols reflects relative magnitude of the $\mathrm{C}_{\mathrm{Exc}}$. The $r$ squared and slope parameters were both significant for the fitted lines $(t$-test, $p<0.01)$.

\section{Discussion}

We have described spatiotemporal neural ensembles in the auditory cortex of an alert cat using a chronic microelectrode array. The microimaging template revealed multiple waves of excitation and inhibition that corresponded to the onset, sustained and offset responses to the stimuli. The waves adhered to a tonotopic representation of sound frequency in AAF, which likely reflects frequency-place properties of the cochlea and underlying auditory structures, as well as the functional neural architecture of the cortex. These sweeping waves in the auditory cortex may play a key role in perception and behavior, analogous to what has been reported recently in the barrel cortex of the rat (Krupa et al 2004, Nicolelis and Ribeiro 2006). 
(a)

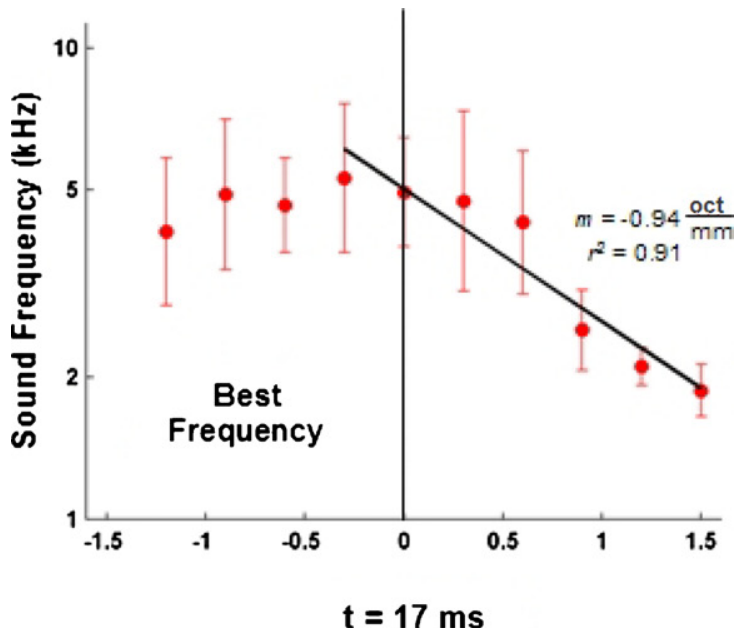

(b)

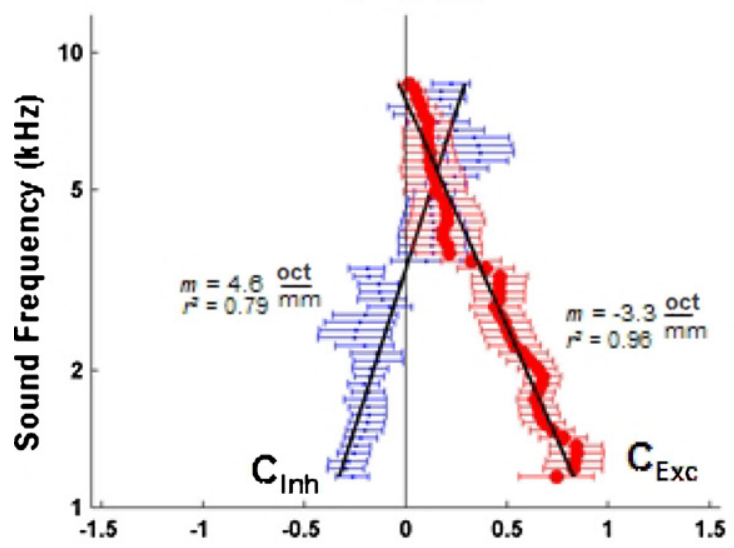

(c)

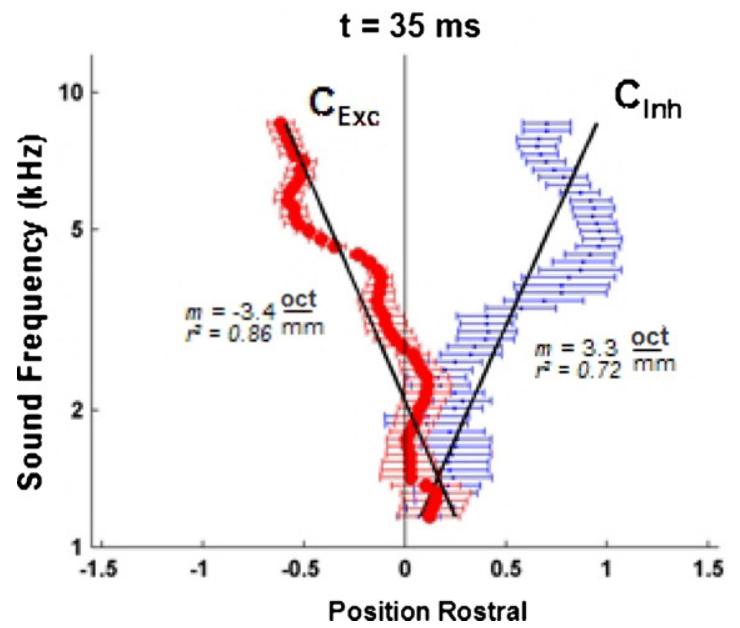

Figure 7. A tonotopic arrangement for the most rostral recording sites $\left(-0.94\right.$ oct $\left.\mathrm{mm}^{-1}, r^{2}=0.91\right)$ is evident in (a), with error bars indicating standard error (SE) across the ten recording sessions. The $\mathrm{BF}$ was defined as the frequency that elicited the largest response based on the mean firing rate during the initial $50 \mathrm{~ms}$ after tone onset. A tonotopic arrangement is also evident in both the inhibitory and excitatory centroids at specific time points (17 and $35 \mathrm{~ms}$ ) in (b) and (c), with horizontal error bars denoting SE. The tonotopic gradient based on $C_{\mathrm{Exc}}$ at $17 \mathrm{~ms}$ was -3.3 oct $\mathrm{mm}^{-1}\left(r^{2}=0.96\right)$; if the spatial peak is used, rather than centroid, the gradient reduces to 1.6 oct $\mathrm{mm}^{-1}$, but with a smaller correlation coefficient $\left(r^{2}=0.79\right)$. Note for consistent comparison, the abscissa and ordinate are the same as in (a), even though the dependent and independent variables are reversed for the regression analysis. The reported slope, therefore, is the inverted slope from the linear regression.

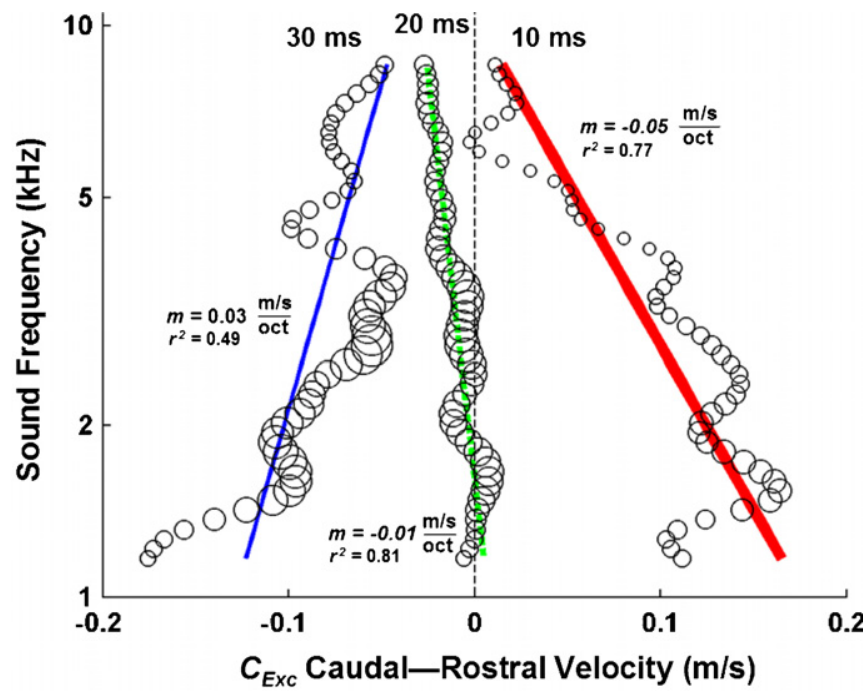

Figure 8. The frequency/velocity relationship for $\mathrm{C}_{\mathrm{Exc}}$ at three time points is conveyed in figure 8 , with a gradient peak at $10 \mathrm{~ms}$ after stimulus onset $\left(m=-0.05\left(\mathrm{~m} \mathrm{~s}^{-1}\right) /\right.$ oct, $\left.r^{2}=0.77\right)$. The magnitude of $C_{\mathrm{Exc}}$ (represented by the size of the open circles) was significant $(Z>3)$ across all frequencies at these times. The $r$ squared and slope parameters were both significant for the fitted lines ( $t$-test, $p<0.01)$. Calculated wave velocities are consistent with horizontal propagation in cat visual cortex (Bringuier et al 1999).

\section{Propagating waves, perception and behavior}

Segregating neural responses into regions of excitation and inhibition (in space and time) emphasizes contrast that may be critical for classifying similar sounds or discriminating frequencies during a listening task. These findings are consistent with enhanced contrast observed in several neural coding and plasticity studies in sensory cortex (e.g., Weinberger (1995), Fritz et al (2003), Miller et al (2002), Ohl and Scheich (1997), (1996), Ohl et al (2001), Witte and Kipke (2005)). Contrast among waves for different stimuli ranging from 1 to $10 \mathrm{kHz}$ suggests that neural coding of different stimuli is a dynamic process that evolves from the site of activation at the scale of milliseconds and microns. Moreover, our results indicate that the frequencyplace representation in auditory cortex was not static, but rather evolved systematically during stimulus presentations. Existing models based on single-cell recording and receptivefield mapping in mammalian auditory cortex suggest that both types of responses contribute to spectral integration and frequency selectivity of auditory cortical neurons, even though their neural pathway (e.g., lateral inhibition) and underlying mechanisms (e.g., neurotransmitters) are likely discrete (Schreiner et al 2000). A theory of spatial coding of multiple sound features in auditory cortex with distinct regions of excitation and inhibition has also been previously proposed (Arbib 1995). The fast propagating waves presented in this initial study support these models of cortical processing and may have particular relevance for frequency discrimination or formant identification during speech.

Other imaging studies have detected propagating brain waves and speculated on their impact. For example, spatial patterns detected with EEG electrodes have long been linked 
to behavior (Lilly and Cherry 1954). These waves may have special relevance to stimulus coding and behavior in sensory cortex that reflect activation and inactivation of distributed pathways (Ermentrout and Kleinfeld 2001). Propagating waves have been thought to encode information about sensory stimuli, especially in visual and somatosensory cortex (Cottaris and De Valois 1998, Derdikman et al 2003, Krupa et al 2004). We report a centroid peak wave velocity of $\sim 0.2 \mathrm{~m} \mathrm{~s}^{-1}$ (figure 8 ) in response to simple tones in the auditory cortex of an awake cat, which is consistent with that reported across the horizontal layers in cat visual cortex based on intracellular recording under anesthesia (Bringuier et al 1999, Chen et al 1997). This value is also consistent with the mean conduction velocity calculated between neurons in vitro (Grinvald et al 1981). Wave speeds were also within the range of in vivo optical imaging studies that detect membrane potential (Grinvald et al 1994). Propagating wave velocities may also provide a baseline as a timing sequence used for chronic cortical microstimulation to restore sensory function (Rousche and Normann 1999). It should also be noted that the waves described in this paper are not necessarily synonymous with evoked dendritic waves detected with surface EEG electrodes. A reduction in the firing rate from a small population of neurons, for example, may lead to a local suppression in activity, but not necessarily a deflection from a baseline EEG or local field potential recordings. Nonetheless, combining this approach with a more global brain imaging tool would enable a more thorough investigation of wave activity at multiple scales. Future work intends to illustrate and contrast different types of cortical waves, detection methods and their underlying mechanisms.

\section{Limitations of microimaging using electrode arrays}

There are drawbacks to using microelectrode arrays to capture spatial and temporal patterns of fast neural activity. First, a reference event (e.g., tone onset) is required to compare a baseline (preceding the event) and evoked (after tone onset) firing rate. The combined real-time multichannel recording system and synchronized event trigger enabled sufficient trial averaging to examine waves at fine spatial resolution $(<1 \mathrm{~ms})$. Because a given channel may produce only five spikes or less during a single tone presentation, trial averaging is necessary to identify significant cortical patterns at high spatiotemporal resolution. Optimal averaging in space (low-pass filtering across recording sites), time (increasing bin size of super PSTHs) and number of trials depends on the constraints of the experiment, acceptable error, assumptions of stationarity and desired resolution. For this experiment, we used 200 repetitions of each stimulus pooled across 10 recording days, a Gaussian spatial smoothing parameter of $\sigma=300 \mu \mathrm{m}$ and $1 \mathrm{~ms}$ bins to describe the cortical patterns.

Second, image quality depends on the number of active recording sites and yield of the recording array. Inactive electrodes (SNR < 2) translate to 'dead pixels' and fail to provide meaningful information. This effect becomes substantial if many electrodes on the array are not functioning; despite sparse sampling, the spatial arrangement of the active recording sites can still convey important information related to the spatial activation pattern, especially when events evolve slowly in brain space. In this case, interpolation and spatial filtering may preserve the underlying pattern of cortical activity, just as filtering out a few bad pixels on a television screen may still preserve the conveyed image or movie.

\section{Other potential applications}

Microimaging using electrode arrays can be combined with other imaging modalities, such as fMRI, PET and EEG, to uncover the relationship between dynamic neural ensembles and the more global measures of fast and slow brain signals. Single-cell electrophysiology has, in fact, been combined with these other methods, such as fMRI (Logothetis et al 2001) and optical imaging using a VSD (Jin et al 2002, Ferezou et al 2006).

From its intricate spatial representation of sensory stimuli, microimaging may serve as an optimal template for control of a cortical prosthetic using intracortical microstimulation (Rousche and Normann 1999) or monitor effects of therapeutic drugs delivered to the brain (Egert and Hämmerle 2002, Williams 2001, Williams et al 2005). The amount of tissue directly affected by drug diffusion or electrical stimulationtypically on the order of at least $100 \mu \mathrm{m}$ from the point of delivery-is consistent with the spatial resolution offered by the microimaging methodology. Although the invasive nature of penetrating electrodes limits their use in humans, neurosurgeons routinely record from single cells during functional brain mapping prior to removal of an epileptic focus or brain tumor (Howard et al 1997, 2000). The microimaging template, when combined with a $3 \mathrm{D}$ stereotactic navigation system, may facilitate and expedite exploratory brain mapping procedures by providing real-time feedback of cortical ensemble activity. Rapid assimilation of neural ensemble activity is also critical for control of a neural or cortical prosthesis. In fact, Cyberkinetics Inc. recently started human trials of a chronically implanted electrode array in motor cortex with the intent of restoring lost motor function.

\section{Conclusions}

We have described initial results of spatiotemporal neural ensembles in the auditory cortex of an alert cat using a chronic microelectrode array. The fast cortical patterns of activity, consisting of both inhibition and excitation, suggest that receptive fields are not static but rather evolve during the stimuli on a millisecond and micron scale. The waves adhered to a tonotopic representation of sound frequency in AAF, which likely reflects frequency-place properties of the cochlea and underlying auditory structures, as well as the functional neural architecture of the cortex. Perhaps, these vibrant cortical microimages from an awake cat invigorate the 'rich mosaic' that Pavlov envisioned almost 80 years ago. 


\section{Acknowledgments}

We appreciate the contributions of Rob Rennaker, Kevin Otto, Ryan Clement and Justin Williams for their help on the project. We also thank Peter Killeen, Eliot Hearst and Jennie Si for insightful comments regarding this manuscript. The research was supported by the National Institutes of Health (R29-DC03070-02), the National Science Foundation (BES9624636) and Defense Advanced Research Projects Agency (MDA 972-00-1-0027).

\section{References}

Arbib M A 1995 The Handbook of Brain Theory and Neural Networks (Cambridge, MA: MIT Press)

Briggman K L, Abarbanel H D and Kristan Jr W B 2005 Optical imaging of neuronal populations during decision-making Science 307 896-901

Bringuier V, Chavane F, Glaese L and Fregnac Y 1999 Horizontal propagation of visual activity in the synaptic integration field of area 17 neurons Science 283 695-9

Buzsaki G 2004 Large-scale recording of neuronal ensembles Nat. Neurosci. 7 446-51

Chen J, Wise K D, Hetke J F and Bledsoe Jr S C 1997 A multichannel neural probe for selective chemical delivery at the cellular level IEEE Trans. Biomed. Eng. 44 760-9

Contreras D and Llinas R 2001 Voltage-sensitive dye imaging of neocortical spatiotemporal dynamics to afferent activation frequency J. Neurosci. 21 9403-13

Cottaris N P and De Valois R L 1998 Temporal dynamics of chromatic tuning in macaque primary visual cortex Nature 395 896-900

Derdikman D, Hildesheim R, Ahissar E, Arieli A and Grinvald A 2003 Imaging spatiotemporal dynamics of surround inhibition in the barrels somatosensory cortex J. Neurosci. 23 3100-5

Egert U and Hämmerle H 2002 Application of the microelectrode-array (MEA) technology in pharmaceutical drug research Sensoren im Fokus neuer Anwendungen ed J P Baselt and G Gerlach (Dresden: Universitätsverlag) pp 51-4

Ermentrout G B and Kleinfeld D 2001 Traveling electrical waves in cortex: insights from phase dynamics and speculation on a computational role Neuron 29 33-44

Ferezou I, Bolea S and Petersen C C 2006 Visualizing the cortical representation of whisker touch: voltage-sensitive dye imaging in freely moving mice Neuron 50 617-29

Fritz J, Shamma S, Elhilali M and Klein D 2003 Rapid task-related plasticity of spectrotemporal receptive fields in primary auditory cortex Nat. Neurosci. 6 1216-23

Goldstein J, Baer T and Kiang N Y-S 1971 The Physiology of the Auditory System ed M B Sachs (Baltimore, MD: National Educational Consultants) pp 133-41

Greenwood D D 1990 A cochlear frequency-position function for several species-29 years later J. Acoust. Soc. Am. 87 2592-605

Grinvald A, Lieke E E, Frostig R D and Hildesheim R 1994 Cortical point-spread function and long-range lateral interactions revealed by real-time optical imaging of macaque monkey primary visual cortex J. Neurosci. 14 2545-68

Grinvald A, Ross W N and Farber I 1981 Simultaneous optical measurements of electrical activity from multiple sites on processes of cultured neurons Proc. Natl Acad. Sci. USA 78 3245-9

Howard M A, Volkov I O, Mirsky R, Garell P C, Noh M D, Granner M, Damasio H, Steinschneider M, Reale R A, Hind J E and Brugge J F 2000 Auditory cortex on the human posterior superior temporal gyrus J. Comp. Neurol. 416 79-92
Howard M A, Volkov I O, Noh M D, Granner M A, Mirsky R and Garell P C 1997 Chronic microelectrode investigations of normal human brain physiology using a hybrid depth electrode Stereotact. Funct. Neurosurg. 68 236-42

Humphrey D R 1979 Electrophysiological Techniques ed D R Humphrey (Bethesda, MD: Saunders) pp 207-59

Jin W, Zhang R J and Wu J Y 2002 Voltage-sensitive dye imaging of population neuronal activity in cortical tissue $J$. Neurosci. Methods 115 13-27

Kim S J, Manyam S C, Warren D J and Normann R A 2006 Electrophysiological mapping of cat primary auditory cortex with multielectrode arrays Ann. Biomed. Eng. 34 300-9

Krupa D J, Wiest M C, Shuler M G, Laubach M and Nicolelis M A 2004 Layer-specific somatosensory cortical activation during active tactile discrimination Science 304 1989-92

Lilly J C and Cherry R 1954 Surface movements of click responses from acoustic cerebral cortex of cat: leading and trailing edges of a response figure J. Neurophysiol. 17 521-32

Logothetis N K, Pauls J, Augath M, Trinath T and Oeltermann A 2001 Neurophysiological investigation of the basis of the fMRI signal Nature $\mathbf{4 1 2} 150-7$

Ludwig K A, Uram J D, Yang J, Martin D C and Kipke D R 2006 Chronic neural recordings using silicon microelectrode arrays electrochemically deposited with a poly(3,4-ethylenedioxythiophene) (PEDOT) film J. Neural. Eng. 3 59-70

Miller L M, Escabi M A, Read H L and Schreiner C E 2002 Spectrotemporal receptive fields in the lemniscal auditory thalamus and cortex J. Neurophysiol. 87 516-27

Nicolelis M A L and Ribeiro S 2006 Seeking the neural code Sci. Am. 68-77

Ohl F W and Scheich H 1996 Differential frequency conditioning enhances spectral contrast sensitivity of units in auditory cortex (field Al) of the alert Mongolian gerbil Eur. J. Neurosci. 8 1001-17

Ohl F W and Scheich H 1997 Learning-induced dynamic receptive field changes in primary auditory cortex of the unanaesthetized Mongolian gerbil J. Comp. Physiol. A 181 685-96

Ohl F W, Scheich H and Freeman W J 2001 Change in pattern of ongoing cortical activity with auditory category learning Nature 412 733-6

Pavlov I 1927 Conditioned Reflexes: an Investigation of the Physiological Activity of the Cerebral Cortex (London: Oxford University Press)

Rattay F 1989 Analysis of models for extracellular fiber stimulation IEEE Trans. Biomed. Eng. 36 676-82

Rousche P J and Normann R A 1999 Chronic intracortical microstimulation (ICMS) of cat sensory cortex using the Utah intracortical electrode array IEEE Trans. Rehabil. Eng. $756-68$

Ruggero M A and Rich N C 1987 Timing of spike initiation in cochlear afferents: dependence on site of innervation J. Neurophysiol. 58 379-403

Schmidt E M 1999 Methods for Neural Ensemble Recordings ed M A L Nicolelis (New York: CRC Press) pp 1-24

Schreiner C E, Read H L and Sutter M L 2000 Modular organization of frequency integration in primary auditory cortex Annu. Rev. Neurosci. 23 501-29

Summerfield Q 1992 Lipreading and audio-visual speech perception Phil. Trans. R. Soc. B 335 71-8

Weinberger N M 1995 Dynamic regulation of receptive fields and maps in the adult sensory cortex Annu. Rev. Neurosci. 18 129-58

Williams J C 2001 Performance of chronic neural implants: measurement, modeling and intervention strategies Bioengineering (Tempe, AZ: Arizona State University)

Williams J C, Holecko M M II, Massia S P, Rousche P and Kipke D R 2005 Multi-site incorporation of bioactive matrices into MEMS-based neural probes J. Neural Eng. 2 L23-8 
Williams J C, Rennaker R L and Kipke D R 1999 Long-term neural recording characteristics of wire microelectrode arrays implanted in cerebral cortex Brain Res. Protoc. 4 303-13

Witte R S 2002 Micro-electrode investigation of neural coding and learning-induced plasticity in auditory cortex Bioengineering (Tempe, AZ: Arizona State University) p 220
Witte R S and Kipke D R 2001 Micro-imaging the auditory cortex: spatiotemporal integration of neural ensemble recording Biomed. Eng. Soc. Fall Meeting (Durham, NC) p P10.6

Witte R S and Kipke D R 2005 Enhanced contrast sensitivity in auditory cortex as cats learn to discriminate sound frequencies Cogn. Brain Res. 23 171-84 